\title{
Second Annual Meeting of the Czechoslovak Microbiological Society
}

\author{
Prague, December 3-4, 1962
}

Abstracts of Communications

\begin{abstract}
Electron microscopy of the surface of yeast protoplasts. O. NeČAS, Department of Biology, Faculty of Medicine, J. E. Purkyně University, Brno
\end{abstract}

Yeast cells are covered with a morphological membrane and a cytoplasm membrane. The morphological membrane is about 5,000 $\AA$ thick and is formed of two layers which can be detected in ultra-thin section. Ultra-thin sections and isolation showed no morphological membrane on the surface of naked protoplasts obtained by means of autolytic enzymes; the protoplasts were enclosed in a cytoplasm membrane only. After isolating the cytoplasm membrane by means of osmotic shock, numerous remnants of cytoplasm were found to be adhering to its inner surface. The cytoplasm membranes were very sensitive to physical influen. ces. Growing protoplasts were likewise enclosed only in a cytoplasm membrane for the whole period of growth. A morphological membrane of the same structure as in normal yeast cells was formed only a few hours before their regeneration into new yeast cells.

Morphological changes induced by ampicillin in cultures of some bacteria. J. VINTIKA, Institute of Pharmacy and Biochemistry, Prague

In experiments with $\mathrm{D} /$-/alphaaminophenylacetamidopenicillanic acid it was found that in some microorganisms L-forms were induced by much smaller doses. This substance, which is active against a great many Gram-negative bacteria, was tested in the following strains: $E$. coli, $P$. morgani, $P$. mirabilis, $S$. marcescens and $R$. leguminosarum. The individual phases of the formation of L-forms were detected by systematic study during cultivation and by changing the cultivation conditions and the culture media, and anomalous features appearing in diffusion tests were explained.

Spore formation in protoplasts of Bacillus megatherium. J. Trpišovská and J. StÁRKa, Department of Microbiology and Genetics, Faculty of Natural Science, Charles University, Prague

Exposure of vegetative cells of Bacillus megaterium 899 to lysozyme produced protoplasts which on further incubation formed spores. In the presence of oxygen the percentage of sporulating protoplasts did not differ from the percentage of sporulating cells in the control. The spores obtained from protoplasts were viable and they did not differ basically from spores obtained from intact. cells in relation to thermoresistance, u.v. resistance and the rate of germination. They had the same refractivity and approximately the same form and size. Vegetative cells which developed from protoplast spores had a normal cell wall and did not differ in any respect from the control vegetative cells. The cell wall thus obviously does not play a fundamental part in spore formation. If it has any direct relation to sporulation at all, it is during the phase before the vegetative cell begins to form granules, which are the first signs of incipient spore formation.

Production of bacteriophage by spheroplasts of a lysogenic strain. L. SošKová, Institute of Biophysics, Czechoslovak Academy of Sciences, Brno

Lysozyme spheroplasts of the lysogenic strain $E$. coli C $6000 / \lambda$ were prepared and spontaneous phage production and phage production after separate and combined induction by u.v. and KCN were determined. The experiments were repeated with intact cells of the same lysogenic strain for comparison. It was found that lysozyme spheroplasts of the given lysogenic strain produced phage both spontaneously and after induction by UV and $\mathrm{KCN}$, but in different amounts than cells of the intact strain. The effect of the two induction agents also differed. The probable explanation of these results is discussed with reference to the findings in the literature.

Induction af bacteriophage and colicine synthesis by u. v. irradiation. J. SMARDA, Department of Biology, Faculty of Medicine, J. E. Purkyner University, Brno

Trradiation of a simultaneously lysogenic and colicinogenic strain of $E$. coli with u.v. rays $(2537 \AA)$ increased phage production 92 -fold and colicine production 40-fold. An attempt was made to determine whether this was due simply to the direct action of radiation on the cells, or whether it was an induction effect caused by chemical changes in the culture medium after u.v. irradiation. Irradiation with a Phillips TUV $15 \mathrm{~W}$ monochromatic u.v. lamp produced no demonstrable changes in the broth leading to induction, even after $60 \mathrm{mi}$ nutes' exposure. An induction effect was, however, 
found in broth irradiated under the same conditions, using large doses, with a Chirana SLU 1 lamp. In this case phage production was increased 4.1-fold (optimal exposure $26 \mathrm{~min}$.) and colicine production up to 2.4-fold (optimal exposure $20 \mathrm{~min}$.). A similar effect was obtained by irradiating the distilled water before preparing and inoculating the broth. In both cases a specific peroxidase and $O$-toluidine reaction demonstrated hydrogen peroxide in the irradiated medium (e.g. in a concentration of $0.000045 \mathrm{~N}$ in broth exposed for $20 \mathrm{~min}$ ). The result corresponded to the curve of the induction effect for different concentrations of hydrogen peroxide. The optimal concentrations were actually higher $0.002 \mathrm{M}$ for phage and $0.0005 \mathrm{M}$ for colicine. Like mutation, phage and colicine synthesis were induced by cultivating the bacteria in u.v. irradiated medium. In both cases the active substance was hydrogen peroxide.

Experiences with the microbiological determination of deoxyribosides and deoxyribonucleic acid. J. Soška, Institute of Biophysies, Czechoslovak Academy of Sciences, Brno

The growth of Lactobacillus (Thermobacterium) acidophilus R-26, which requires the presence of deoxyribosides, was studied in relation to the presence of certain ingredients in the culture medium or test solution and in relation to the type of deoxyriboside present. The most satisfactory conditions for the microbiological detection of deoxyribosides in biological material were also determined. The determination of low molecular weight deoxyribosides and deoxyribotides in $E$. coli is cited as an example.

$X$-ray sansitivity of Bacterium coli during starvation and arrested nucleic acid synthesis. B. Lrška, Institute of Biophysics, Czechoslovak Academy of Sciences, Brno

The radioresistance of $\mathrm{X}$-irradiated starving cells of Bacterium coli was greater than that of bacteria in the middle of the log phase of growth. Resistance was also raised after stopping nucleic acid synthesis in the auxotrophic strain Bacterium coli $B$, which requires the pyrimidine base of nucleic acid. The results were supplemented by an analysis of the nucleic acid and protein concentration in irradiated bacteria and were compared with the data in the literature on the influence of the synthesis and amount of protein and droxyribonucleic acid on radioresistance.

Determination of the activity of certain enzymes of $C$ motabolism in green and depigmented cells of Euglenx gracilis. L. Drobrica and L. Ebringer, Department of Technical Microbiology and Biochemistry, Faculty of Chemistry, Slovak Technical College, Microbiology Section, Department of Plant Physiology, Faculty of Natural Science, Bratislava
Loss of the ability of Euglena gracilis to synthesize chlorophyll, caused by streptomycin, was long regarded as a specific property of this antibiotic only. It was found that other antibiotics of the streptomycin and erythromycin group also have a similar effect. The Ioss of chlorophyll is irreversible, i.e. the euglenae are permanently heterotrophic. Aldolase, glycerinaldehyde-phosphate-dehydrogenase, phosphoglycerate-kinase, pyruvate-kinase, malate-dehydrogenase and glutamate-oxalacetatetransaminase activity were determined in extracts of green and streptomycin- or erythromycin-depigmented cells of Euglena gracilis obtained by a freezing process. Glutamate-dehydrogenase, glutamate-pyruvatetransaminase and lactate-dehydrogenase (LDH) activity, which functions in combination with DPN, was not demonstrated. It is interesting to note that Euglena gracilis utilizes lactate as a source of carbon. The authors express the hypothesis that the LDH of Euglena gracilis cells is of a flavoprotein character and that the cells do not therefore require DPN. The significance of differences in the activity of certain enzymes in green and depigmented cells and the significance of the findings on the nature of $\mathrm{LDH}$ are discussed with reference to the systematic classification of Euglena gracilis.

Localization of amino acid oxidases in the bacterial cell. M. V. Nermut, Department of Biology, Faculty of Medicine, J. E. Purkyně University, Brno

A substrate was found for the reduction of potassium tellurite by cells of Proteus vulgaris (this also applies to other species of bacteria). The substrate consists of amino acids, e.g. for Proteus vulgaris lysine, ornithine, methionine, leucine, glutamic acid, etc. The enzymatic nature of reduction was again demonstrated. The position of the tellurium microcrystals was studied by electron microscopy, using the ultra-thin section technique. Amino acid oxidases were found to be localized in the vicinity of the cytoplasm membrane, and also in the cytoplasm.

The question of regulation of protease formation and activity in the bacterial cell. J. CHALOUPKA,

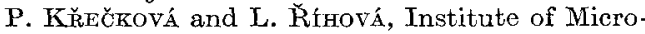
biology, Czechoslovak Academy of Sciences, Prague

The formation of proteolytic enzymes by Bacillus megaterium is inhibited if amino acids are present in the culture medium. Repression by amino acids occurs both in a growing culture and in a non-growing culture resuspended in fresh medium. The same amino asid concentration has little or no effect on the formation of intracellular protease. Synthesis of this enzyme, however, is dependent on growth of the cells. The activity of intracellular protease in cell homogenate is reduced by about $40 \%$ by an endogenous inhibitor. The nature of this inhibitor 
and the relationship of the given concentration of amino acids in a bacterial pool to protease activity are discussed.

Induced urease synthesis in Aerobacter aerogenes. H. Honzoví and J. Stárka, Department of Microbiology and Genetics, Faculty of Natural Science, Charles University, Prague

Diauxie was observed during the growth of Aerobacter aerogenes in mineral medium containing ammonium sulphate and urea. When the ammonium sulphate is exhausted, growth stops and further growth, at the expense of urea, does not occur until after a lag lasting about the time of one generation period, i.e. 45 minutes. Cells which utilize ammonium-bound nitrogen display no urease activity. Urease is synthesized rapidly during the lag phase. Its activity per cell remains constant for only one generation, however. When further growth commences, synthesis of the enzyme is again markedly inhibited by the ammonium ions which accumulate from hydrolysed urea. Urease synthesis is thus regulated by a mechanism in which urea acts as the inductor and ammonium ions as the corepressor.

Disintegration of bacterial cells. P. Novotry, Laboratory for Medical Microbiology, Charles University, Prague

A simple apparatus was constructed for disintegrating cells by means of glass beads, permitting work at controlled temperatures. In the test strain of bacteria disintegration by at least $99 \%$ took $1-2 \mathrm{mi}$ nutes, while in Mickle's ball mill the same final effect, in the same strain, took 45 minutes. The quality of the resultant preparations is discussed.

Effect of iodoacetate on the dehydrogenase activity of rough and smooth forms of yeasts. L. SILHÁNková, College of Chemical Technology, Prague

The causes of differences in the sensitivity of aerobic metabolism of ethanol and acetate to the inhibitory effect of iodoacetate were determined in smooth forms of yeasts and in rough forms produced by spontaneous dissociation of yeast cultures. The dehydrogenation activity of whole cells of smooth forms was inhibited by a $10^{-4} \mathrm{M}$ iodoacetate con. centration (by about $20 \%$ ) in the presence of ethanol and acetate, while in rough forms it was not affected. The iodoacetate sensitivity of dehydrogenation of ethanol by cell-free preparations was the same for both forms of yeasts, althougl. where were differences between their dehydrogenases (e.g. in their behaviour in borate buffer). It is assumed that differences exist between the two forms of yeasts in the permeability of the cell membrane for iodoacetate, or in the effect of iodoacetate on the uptake of ethanol or acetate by the cells. Further evidence of differences in the functional properties of the cell membranes of the two forms of yeasts is also to be found in the need to raise the $\mathrm{pH}$ above the optimum for dehydrogenation of ethanol in whole cells of smooth forms in order to be able to detect any dehydrogenase activity.

The amylolytic system of Endomycopsis capsularis. H. EBertová, Institute of Microbiology, Czechoslovak Academy of Sciences, Prague

The ability of 170 species of maltose-assimilating yeasts to break down starch and convert glucose from the $\alpha(1-4)$ to the $\alpha(1-6)$ glucoside bond was dutermined. The yeasts which decomposed starch best were Endomycopsis capsularis, $E$. fibuliger, $E$. chodati, Candida japonica, Lipomyces starkeyi, Sporobolomyces pararoseus and Bullera alba. The genera Schizosaccharomyces, Endomycopsis, Lipomyces and the species Pichia polymorpha, Mycotorula japonica and Ashbya gossypii were found to have the greatest transglucosidase activity. The amylolytic system of Endomycopsis capsularis, released into the medium, contains amyloglucosidase, 1-2 enzymes of the $\alpha$-amylase type and an enzyme capable of transglucosidation to the $\alpha(1-6)$ glucoside bond. The optimum $\mathrm{pH}$ for these enzymes is about 4.5 and the optimum temperature $40-45^{\circ} \mathrm{C}$. Amylase and glucosidase are most stable at $\mathrm{pH} \quad 5-7$ and amyloglucosidase at $\mathrm{pH} \quad 4.3-8$. The rate of production of glucose by amyloglucosidase and the rate of formation of dextrins and oligosaccharides by amylase falls with the molecular weight of substrates of the amylose series. Oligosaccharides of the iscmaltose series are broken down by these two enzymes at a 100-1,000 times slower rate. The rate of hydrolysis of saccharides of the maltose and isomaltose type by transglucosidase is in inverse proportion to their molecular weight. Isomaltose is hydrolysed twice as slowly as maltose. Under submerse cultivation conditions, Endomycopsis capsularis produces a larger amount of transglucosidase at the outset of the log phase and amyloglucosidase and amylase after growth is complete. At pH 3-4 more amyloglucosidase is produced, at about $\mathrm{pH} 6$ more amylase is formed. Differences in the relative proportion and concentration of the enzymes in relation to the carbon source (glucose, maltose, soluble starch) are negligible.

Isolation and propertues o yeast ribosomes. V. Ltebl, Ch. Singh, M. StTrna dová, Institute of Microbiology, Czechoslovak Academy of Sciences, Prague

Ribosomes were isolated from Torula utilis and characterized. The cells were broken up in Nossal's ball mill, using glass beads (ballotina No. 14). The proportion of broken cells was ascertained by determining the amount of nucleic acid released into the supernatant fluid and the amount remaining in the sediment. Disintegration was more effective 
(about $90 \%$ ) with yeasts which had been frozen for several hours (in fresh yeasts it was about $60 \%$ ). Ribosomes isolated from homogenate by differential centrifugation showed no signs of injury. The sedimentation constant $80 \mathrm{~S}$, the nucleic acid and protein concentration and the behaviour of the ribosomes on DEAE cellulose were determined and were compared with a suitable artificial model ribonucleoprotein system. Only part of the ribonucleoprotein was released under these conditions. The aggregation and breaking down of the ribosomes and the types of bonds formed were also studied. The enzymes bound in the ribosomes were found to include ribonuclease and protease. Some of the bonds between proteins and nucleic acids in the ribosomes were very strong (chemical, hydrogen bonds, etc), while others, evidently of an electrostatic nature, etc., were weaker and were dependent on the character of the medium. The structure of the ribosome was complex and probably consisted of sever a layers.

Effect of ionizing radiation on the biological properties of Mycobacterium tuberculosis. Z. ProCHÁzKa and M. PAvLAS, Institute of Veterinary Medicine, Brno-Medlánky

When testing the effect of $\gamma$-radiation in doses of $100,000-1,000,000 \mathrm{r}$. on the biological properties of Mycobacterium bovis, a decrease was found in its allergogenic properties in guinea-pigs after irradiat. ing it with a dose of $500,000-1,000,000 \mathrm{r}$. No effect on the vitality of the mycobacteria was found after irradiation with $100,000-200,000 \mathrm{r}$. After irradiation with $300,000-600,000$ r., the number of colonies decreased progressively as the radiation dose increased. After irradiation with 700,000-1,000,000 r. the mycobacteria were completely devitalized. A marked decrease in virulence was demonstrated in guinea-pigs after a dose of 400,000-500,000 r. Mycobacteria irradiated with a dose of 600,000 to $1,000,000$ r. produced no tuberculous changes in any of the experimental animals (not even at the site of inoculation).

Effect of different freezing and lyophilization techniques on the metabolic activity of Mycobacterium tuberculosis, strain BCG. I. DROBNIcová, Institute of Tuberculosis, Bratislava

The author describes the results of experimente investigating the effect of different freezing and lyophilization techniques, with reference to changes in the number of living organisms (cultivation) and in metabolic activity $\left(\mathrm{Q}_{2}, \mathrm{Q}_{\mathrm{CO}_{2}}, \mathrm{RQ}\right.$ on Sauton medium, endogenous respiration, glycerin-dehydrogenase activity). She studied the significance of the number of degrees. (down to $-70^{\circ} \mathrm{C}$ ) and time of freezing of the culture and of the lyophilization technique, maintaining a vacuum of $10^{-2} \mathrm{~mm}$. $\mathrm{Hg}$, without a source of heat and with various heating arrangements controlled by means of a thermocouple built into the lyophilizer (the lyophilization ampoule). The best results were obtained with cells frozen in a lactose medium at $-20^{\circ} \mathrm{C}$, lyophilized for 10 hours with a controlled heating programme giving a steady increase in temperature from $-20^{\circ}$ to $+30^{\circ} \mathrm{C}$.

Biochemical significance of the molybdenate test for the identification of yeasts and yeast-like microorganisms. A. KockovA-KRatochvíLOVA and A. Vojtkova, Microbiology Laboratory, Institute of Chemistry, Czechoslovak Academy of Sciences, Bratislava

It is known that some yeast-like microorganisms become coloured in media containing salts of tellurium, selenium or mo ybdenum. The results of this test were studind in different strains of four types of yeasts cultivated by the method used in the fermentation of maltose and sucrose. A correlation was found between the type of yeast and the result of the molybdenate test. This emphasizes the significance of the molybdenate test for the identification of yeasts and yeast-like organisms. An attempt was made to explain this reaction on the basis of the manner in which the given strain metabolized sucrose. In analytical chemistry the molybdenate test is used to demonstrate sucrose in the presence of acids; in this case, after inversion, the fructose component of sucrose gives molybdenum blue more rapidly and intensively than the glucose component. The utilization of sucrose by different types of yeasts was therefore studied in metabolized medium containing sucrose by analysing the sugars and by determining organic acid formation, both quantitatively and by paper chromatography.

Notes on the taxonomy of the Serratia group. J. SEDLÁK and V. DLABą, Department of Microbiology, Faculty of Hygiene, Charles University, Prague

The authors studied over 70 strains of the Serratia group, including original collection cultures and strains isolated from human pathological material. The biochemical properties of cultures from collections designated as $S$. marcescens, $S$. indica and $S$. plymouthica were compared. The purpose of the experiments was to attempt serological differentiation of the given series. Biochemically the series formed a markedly homogeneous group, irrespective of species. According to their $O$ antigen interrelationships, these species can be divided into ten serologically quite sharply defined groups. A given antigenic relationship, based on the existence of common partial antigens (antigen minor) was demonstrated between groups $03,04,09$ and 010 . The experiments confirmed the recently expressed view that the genus Serratia is basically a monotypical gromp with $1-2$ biochemical variants. Serotyping was found to be a suitable taxonomic method and when elaborated further it will prove 
quite as useful as serotyping of the other members of the Enterobacteriaceae family.

Phosphatase activity of staphylococci. V. DLABAč, Department of Microbiology, Faculty of Hygiene, Charles University, Prague

A method for quantitative determination of the phosphatase activity of microorganisms was elaborated, using the sodium salt of phenolphthaleindiphosphoric acid as substrate. This method gave basic information on some of the properties of the acid phosphatase (phosphomonoesterase) of staphylococci. The dependence of phosphatase formation on different cultivation conditions and the stability of this property during storage of the strains under laboratory conditions were also studied. Comparison of the phosphatase activity of over 300 strains of staphylococci showed marked differences in the ability of individual strains to form acid monoesterase. The lowest phosphatase activity values were observed in coagulase-negative staphylococci, while the average values in the group of coagulase-positive staphylococei were higher. The findings are compared with those of other authors.

Study of the relative specificity of mutagens in the induction of auxotrophic mutants. J. NEČÁsEk, Department of Microbiology and Genetics, Faculty of Natural Science, Charles University, Prague

Intergenic differences in the mutagenic effect of u.v. light and $\mathrm{N}$-ypperite were studied in the strain Corynebacterium $s p$. VUA 9366, which is important from the aspect of the possibility of industrial biosynthesis of amino acids. Auxotrophic mutants were obtained by the penicillin method (with the cooperation of J. Plachý). Phenotyping of mutations with a delay in manifestation was carried out on liquid medium. The effect of the mutagens was evaluated not from the incidence of mutants, but of the rate of mutation, assuming that mutants of the same phenotype are formed by multiplication of one mutant cell. Statistically significant differences were found between the numbers of mutations determining dependences on arginine or citruline, on arginine or citruline or ornithine, on valine and isoleucine and on the components of hydrolysate of nucleic acid. These results are in agreement with the findings of Nakayama and Kinoshita ( $\mathrm{J}$. gen. appl. Microbiol. $7: 155,1961$ ), who worked with a similar strain, and contradict the views of Westergaard (Abh. Deutsch. Akad. Wiss. 1 : 116, 1960).

Effect of X-rays on variability of Streptomyces noursei. F. PALEర̌Ková, Institute of Antibiotics, Roztoky near Prague

The effect of different doses of X-rays on the variability of cycloheximide production in a nyst- atin-producing strain of Streptomyces noursei is described. Doses of $25,000,50,000,100,000,200,000$ and $400,000 \mathrm{r}$. were selected to determine the killing effect. For studying the effect on production variability, doses of 100,000 and $200,000 \mathrm{r}$. were used. A series of specimens isolated from each experiment was evaluated by cultivation on a shaker apparatus in two independent experiments. The effect of $\mathrm{X}$-rays was compared with the effect of $u . v$. radiation and N-methyl-dichlordiethylamine $\mathrm{HCl}$. Exposure for u.v. radiation was 16 seconds (survival $0.5 \%$ ). $\mathrm{N}$-methyl-dichlordiethylamine $\mathrm{HCl}$ was administered in $0.01 \mathrm{M}$ concentration for $30 \mathrm{mi}$ nutes (survival $0.6 \%$ ). The variance of average production by the various specimens in series treated with a mutagenic factor was compared with variance of production in the relevant control series. The value $\mathrm{F}$ was used to test whether these differences were statistically significant (for $\mathbf{P}=$ $=0.05$ and $P=0.01$ ). Under the given experimental conditions, only light was found to increase the variability of cycloheximide production by Streptomyces noursei significantly.

The role of thiamine in the biosynthesis of phenylacetylcarbinol in yeasts. O. HANČ and H. BEČvářová, Institute of Pharmacy and Biochemistry, Prague

According to Neuberg, optically active phenylacetylearbinol is formed, together with benzyl alcohol, during fermentation of a saccharide medium by Saccharomyces cerevisiae in the presence of benzaldehyde. A series of parallels can be drawn between the biosynthesis of acetoin and of this fatty-aromatic ketol. In the former case pyruvic acid and acetaldehyde act as precursors, in the latter pyruvic acid and benzaldehyde. The most important factor is the presence of yeast carboxylase, which concerts pyruvic acid to activated acetaldehyde. It was found that thiamine alone stimulated the formation of $\mathrm{CO}_{2}$ in fermentation of a sugar and pyruvic acid medium by yeasts, but that, in the given concentrations, which were $10-100$ times higher than the normal level in yeasts, it did not directly influence the increase in the biosynthesis of phenylacetylcarbinol. On incubating thiamine alone with pyruvic acid and benzaldehyde in a mildly alkaline medium, phenylacetylcarbinol was formed without the presence of enzymes, however, while in incubation with acetaldehyde instead of pyruvic acid, the $\beta$-ketol isomer acetylbenzoyl was formed. According to Mizuhara, nonenzymatic acetoin is also formed in a similar manner.

Trypacidin - a new antibiotic active against Trypanosoma cruzi. J. BALAN, L. EBringer, P. NĚMEC and Š. Kovác, Czechoslovak Academy of Sciences, Institute of Biology, Slovak Academy of Sciences, Department of Technical Microbiology and Department of Microbiology, Faculty 
of Natural Science, Comenius University, Bratislava, Department of Organic Chemistry, Slovak Technical College, Bratislava

A method of screening antibiotics was evolved, with special reference to the isolation of substances active against protozoa. A new antibiotic active against Trypanosoma cruzi was isolated by this method from a sample of soil from China. The antibiotic was named Tripacidin. In vitro it is effective against Trypanosoma cruzi in a concentration of $6-8 \mu \mathrm{g} . / \mathrm{ml}$.; it is ineffective against fungi and $\mathrm{G}+$ and $\mathrm{G}$ - bacteria. The screening method and some of the biological, chemical and physical-chemical properties of the new antibiotic are described.

Biosynthesis of cycloheximide and similar substances. J. SPfžEk, I. MÁLEK, Z. VANĚK, M. PƯ ŻA and M. Vondrá̌rer. Institute of Microbiology, Czechoslovak Academy of Sciences, Prague

The biosynthesis of cycloheximide and similar compounds produced by the actinomycete $S$. noursei is resolved by means of an isotope technique and methods of microbiological genetics. Degradation techniques facilitated measurement of the radioactivity of the individual carbons of the dimethyl. cyclohexane nucleus and the glutarimide ring and formation of a basic concept of the structural units of cycloheximide. Biogenetic relationships and the possibilities of regulating the biosynthesis of cycloheximide, streptovitacins and actinophenol are studied through the influence of mutagenic factors on the formation of mutants of $S$. noursei with altered production of these substances. The relationship of cycloheximide synthesis to the synthesis of clavatol by Aspergillus niger and of sorbicillin by Penicillium chrysogenum is discussed.

Preparation of 6-aminopenicillanic acid by enzymatic decomposition of penicillin. Z. HošxLEK, A. B̈̌Ě̌KA, J. SlezÁK, B. SikyTA and M. HerouD, Institute of Antibiotics, Roztoky near Prague

Only $5 \%$ of 400 test strains of Escherichia coli obtained from collections and by isolation from natural material possessed active acylase, which breaks down benzylpenicillin to 6-aminopenicillanic and phenylacetic acid. Marked acylase activity was found only in the strains $E$. coli ATCC 9637, ATCC 11105 and $M$ 200. Strains isolated from natural material (stools) were all inactive. Acylase formation depends primarily on the conditions of cultivation of $E$ coli. Its synthesis requires a rich, complex medium containing peptone and corm steep. The presence of phenylacetic acid in the medium stimulates activity, though not with every strain. Growth on media containing sugars as the carbon source was intensive, but the cells did not break down penicillin. The enzymatic decomposition of penicillin is carried out with 10 -hour cells separated from the nutrient medium and suspended in aqueous benzylpenicillin solution, adding butyl acetate, which alters the permeability of the plasma membrane and causes autolysis of the cells. The optimal temperature for acylase activity is $43^{\circ} \mathrm{C}$ at pH 7.3. During decomposition the pH falls as a result of the release of phenylacetic acid. The decrease in the $\mathrm{pH}$ is regulated by constantly adding alkaline solution. When the penicillin has been decomposed the cells are centrifuged off and can be used for several more decomposition experiments. The resultant 6-aminopenicillanic acid solution is condensed and after acidification the substance crystallizes out of the solution.

Experiences with the preparation of $\mathrm{x}$-aminopenicillanic acid. M. KULHÁNEK and M. TADRA, Institute of Pharmacy and Biochemistry, Prague

Using a cellular suspension of a strain of bacteria isolated from natural material, benzylpenicillin was hydrolyzed in water to which toluene had been added. The resultant 6-aminopenicillianic acid was isolated in crystalline form, giving over $60 \%$ of the theoretical yield with 0.5 and $1 \%$ penicillin solution and up to $45 \%$ with $2 \%$ penicillin solution (in each case related to the amount of penicillin). The cellular suspension could be used several times, the highest yield being obtained on the second or third occasion.

\section{Biosynthesis of bacitracin. A. Bर̌EČKA, Institute. of Antibiotics, Roztoky near Prague}

After finding that bacitracin synthesis is directly associated with spore formation, protoplasts or washed cells producing the strain Bacillus subtilis var. licheniformis were generally used for studying the conditions. It was found that leucine, isoleucine, histidine, asparagine, ornithine and cysteine were important for the biosynthesis of bacitracin and that it was stimulated by glucose and by a "peptide factor" in soya flour (Snoke, J. E., J. Bacteriol. 80 : 552, 1960). As distinct from these experiments, the author studied the conditions of the biosynthesis of bacitracin in cultivation of the producer strain on medium containing complex sources of nutrients and in the presence of the optimal conditions for formation of the antibiotic (summed up in the paper). The experiments showed that, in the presence of an adequate concentration of soya flour in the fermentation medium, the addition of amino acids contained in the bacitracin molecule had a negative effect on synthesis of the antibiotic and that the formation of bacitracin was markedly inhibited by adding corn steep as a complex source of amino acids or by substituting soya flour hydrolysate for soya flour. It can be assumed that amino acids are not the precursor of bacitracin, since a high amino acid concentration in the fermentation medium inhibits production of the antibiotic, and 
that the precursor is probably the "peptide factor", which will be studied in further experiments.

\section{Microbiological transformation of some pyrimidine} derivatives. A. CAPEK and M. TADRA, Institute of Pharmacy and Biochemistry, Prague

A biphasic fermentation technique was used to study transformation processes in the molecules of pyrimidine derivatives by means of microorganisms, i.e. cultivation took place in nutrient medium and when this was complete the biomass was separated to distilled water containing the test pyrimidine derivative. Actual transformation took place in this medium, under submerse conditions. A strain of E. coli (ribosidation) and Aspergillus nidulans (hydroxylation) were used for the transformation of pyrimidine derivatives. Ribosidation of pyrimidine derivatives is dependent on the constitution of the heterocycle and on the presence of the relevant saccharide. Among the variously substituted test pyrimidines, only 6-azauracil was capable of ribosidation by $E$. coli. In formation of the ribose of 6-azauracil by this method, glycerin, dihydroxyacetone, D-ribose, D-xylose, L-arabionose, L-rhamnose, D-sorbitol, D-mannitol, D-glucose, D-mannose, D-galactose, D-fructose, D-tagatose, lactose, maltose and trehalose and gluconic acid can be used as a source of sugar. If L-erythritol, D-arabitol, L-arabitol, D-xylitol, adonitol, D-arabinose, dulcitol, L-sorbose, 2-keto- or 5-keto-gluconic acid, sucrose or cellobiose are used as the sugar source, the above method never produces ribosidation of the 6-azauracil molecule. In study of hydroxylation of derivatives of the pyrimidine molecule, hydroxyl formation was demonstrated only in cases in which C2 was not occupied by another substituent.

Study of the biosynthesis of 6-azauridine. V. MATELOVÁ, Institute of Antibiotics, Roztoky near Prague

A technique for the biotransformation of 6 -azauracil to 6 -azauracil riboside, with Escherichia coli $B$ as the control strain, on synthetic medium was elaborated. The optimal increase in the inoculum was $10^{\mathrm{to}}$ to $1.8 \times 10^{10}$ cells $/ \mathrm{ml}$. and optimal growth of the culture on adding 6 -azauracil was $10^{20}$ cells $/ \mathrm{ml}$. Transformation took $7-9$ hours and $80-95 \%$ of the added azauracil $(0.04 \%)$ was transformed. An increase in the glucose concentration in the medium and enrichment of the medium with yeast extract, soya flour, corn steep, or humin substances, ete, had a favourable effect on ribosidation. Medium containing ammonium acetate was suitable for transformation. Sodium nitrate was an unsuitablo source of nitrogen. Meat extract stimulated ribosidation but did not increase the yield. The optimal $\mathrm{pH}$ for ribosidation was $6.5-7$. On the above semisynthetic media $85-95 \%$ of the 6 -azauracil $(0.08 \%)$ was transformed to 6 -azauracil riboside. The course of ribosidation was not influenced by the amount of inoculum, the rate of aeration or the dose of 6-azauracil. When studying the ability of bacteria, proactinomycetes, actinomycetes, yeasts and fungi to mediate ribosidation of 6 -azauracil it was found that $70 \%$ of the test strains were capable of effecting transformation. On increasing the dose of 6-azauracil, some bacteria, actinomycetes and fungi displayed higher ribosidation capacity than the control strain of Escherichia coli $B$. Yeasts were either not capable of transformation at all, or only to a very limited degree.

\section{Microbiological dehydrogenation of $3 \beta-O H \vartheta^{5}$ unsaturated pregnane derivatives. J. Protiva, V. Schwarz and K. SyHora, Institute of Natural Medicinal Substances, Prague}

Reports on microbiological dehydrogenation of the $\vartheta^{5}-3 \beta$-OH system to the $\vartheta^{4} 3$-keto system were published as early as 1938 , with a description of the effect of different microorganisms on cholestane, androstane or pregnane derivatives. In a detailed study of this reaction, using different species of the genus Flavobacterium and 21 -acetoxylated derivatives of the pregnane series, the authors found that two of the four test species effected the required transformation without hydrolysing 21 -acetoxyl. Evidence of the absence of the relevant hydrolytic enzyme system in these flavobacteria is provided by the finding that they do not attack such a labile ester bond as formyl. The other two species of Flavobacterium oxidize 3-formoxy-21-acetoxy- $\vartheta^{5}$ -pregnene-17 $\alpha$-ol-20-one, giving rise to $\vartheta^{4}-17 \alpha-21$. -diol-3,20-dione (Reichstein's steroid S) and thus normally hydrolyse any ester bonds present.

Biosynthesis of lysine and glutamic acid. M. HERotn, Institute of Antibiotics, Roztoky near Prague

In the first phase of their experiments, a team of workers at the Institute of Antibiotics concentrated on the isolation of suitable bacterial cultures permitting isolation of large amounts of the above amino acids, which are nutrionally important in livestock production. Their methods, which were similar to those used by Japanese workers (Nakayama, K. et al., J. gen. appl. Microbiol. $7: 41$, 1961) were based on the assumption that auxotrophic mutants requiring homoserine for growth and obtained from a culture, which, in the prototrophic state, produces glutamic acid, would be capable of synthosizing lysine. In the strain Corynebacterium sp., VÚA 9366 , which was isolated by the author's team and which produced glutamic acid, the administration of mutagens and the penicillin method (Davis \& Lederberg) induced a number of mutants which produced substantial amounts of lysine in submerse fermentation. The basic factors determining biosynthesis of lysine are discussed, with special reference to the influence of the various ingredients of the fermentation medium and the technological conditions of fermentation. 
The results will provide a reliable basis for economical industrial production of the above amino acids.

Antimicrobial activity of isothiocyanates derived from acridine and benzacridine. P. NEMEC, P. Kristián, L. Drobnica, K. Antoš and A. Holka, Faculty of Chemistry, Slovak Technical College, Bratislava

The results of determination of the antibacterial and anti-yeast activity of isothiocyanate acridines (ITCA), including 2-ITCA, 3-ITCA, 4-ITCA, 1-nitro-5-ITCA, 2-nitro-5-ITCA, 2,8-di-ITCA, 10-ITC-benz(c)-acridine, 9-ITC-benz(a)-acridine and the corresponding amino derivatives are described. Substitution of the ITC group for the amino group on the acridine nucleus usually lowers antibacterial activity (at $\mathrm{pH} 7.4$ ) (this is attributed to a decrease in the reactivity of the nitrogen heteroatom) and markedly raises its antiyeast activity, which is proportionate to the chemical reactivity of the -NCS group. Comparison of the quantities characterizing chemical reactivity (the constants of the rate of addition of $\mathrm{OH}$ ions to the NCS group) and of the pCa values of ITCA shows that reactivity increases in the order 3-, 4-, 2- and 5-ITCA, which is in agreement with concepts on the electron structure of these substances. From $\operatorname{LD}_{5 n}$ values (intraperitoneal administration in mice) it was concluded that the toxicity of some ITCA derivatives is lower than that of the corresponding amino acridines.

Mechanism of the effect of tetracycline antibiotics. 6-phosphatase activity of staphylococci and the effect of oxytetracycline. V. KRČMERY and M. FERENČr, State Veterinary Institute and Veterinary Inspection Station, Bratislava

The glycerophosphate and nitrophenylphosphate method was used for quantitative study of the phosphate activity of staphylococci. Both substrates were the only nutrient medium for staphylococcal strains. Phosphatese activity was studied in highly sensitive strains isolated from pathological material and in polyresistant hospital staphylococci. Differences in phosphatase activity were found in both groups. Oxytetracyaline (OTC) in subst. inhibited the phosphatase activity of polyresistant and OTC-resistant strains and of three OTC-sensitive strains of Brucella melitensis characterized by high phosphatase activity. The significance of the finding of inhibition of phosphatase for the mechanism of the effect of OTC is discussed.

Dynamics of the change in the sensitivity of micro. organisms to some antibiotics. F. VÝmoLA and Z. JEDLǏ̌Ková, Institute of Epidemiology and Microbiology and Department of Microbiology and Epidemiology, Post-graduate Medical Institute, Prague

Faviform variability of Triehophyton gypseum.
M. Hejtmángová-Uhrová, Department of Bio. logy, Faculty of Medicine, Palacký University, Olomouc

Minimal concentrations of the antimycotic phenylmercuridibutyl-naphthyl sulphonate produce faviform changes in Trichophyton gypseum var. asteroides, which have the character of non-fixed modifications. In its form and mauve pigmentation the faviform variant resembles colonies of Trichophyton violaceum. It retains its pathogenicity and produces the familiar picture of tinea. The effect of the antimycotic on the culture was not manifested until the dermatophyte was recultivated, when morphologically anomalous cultures were isolated. Cultures corresponding to Trichophyton gypseum var. Kaufmann.Wolfii were always reisolated from the lesions produced by the faviform variant. Certain changes in the physiology of this dermatophyte also occurred in association with the morpho. logical change. Unlike normal colonies, the faviform variant was capable of assimilating inorganically bound nitrogen sources $\left(\mathrm{NO}_{3}, \mathrm{NH}_{4}\right)$. In this respect it resembles Trichopton violaceum.

Use of enzymatic inhibitors in the study of microbiological formation of humus. Sodium azide. B. Novák and S. Franklová, Central Research Institute for Plant Production, Prague-Ruzynĕ

When studying humification under model conditions, $\mathrm{NaN}_{3}$ was used as an inhibitor of oxidative phosphorylation. It was demonstrated that a block of this enzymatic system also inhibited the formation of humins, particularly humic acids, without affecting the mineralization of organic substances. The results provide further evidence showing that the formation of humus is a synthetic process and that it is therefore markedly influenced by systems releasing and transmitting energy. The larger the amount of humins to be synthesized "de novo", the more pronounced is the influence of these systems.

The biology of geophilic dermatophytes. M. HEJTMÁNEK, Department of Biology, Faculty of Medicine, Palacký University, Olomouc

The following species of dermatophytes have been isolated from soil in Czechoslovakia: Keratinomyces ajelloi, Microsporum gypseum, M. cookei and Trichophyton terrestre. The largest numbers of specimens were isolated from localities regularly frequented by livestock. The keratinolytic activity of $K$. ajelloi was greater than that of $M$. gypseum. These fungi evidently act as natural destroyers of keratinous remnants in soil. When cultivated on Sabouraud glucose medium all the above species became pleomorphous. The ability of pleomorphous cultures to assimilate inorganic nitrogen sources was significantly higher than that of the initial cultures. Under conditions similar to natural conditions these species formed kleistothecia, with an abundance of 
normally developed sporangia containing eight acrospores. The main differences between the species were in the morphology of the peridium layer and in the pigmentation of the kleistothecia. Comparison of cultures of Trichophyton terrestre Durie et Frey and of Ctenomyces trichophyticus Szathmáry showed that they were identical.

Inhibition of virus multiplication by 5-arylpyrimidine derivatives. B. RADA, D. BLAškovič, Z. BUdǐšnskÝ and Z. Peřina, Institute of Virology, Czechoslovak Academy of Sciences, Bratislava and Institute of Pharmacy and Biochemistry, Prague

The inhibitory effect of 5-arylpyrimidines was studied by the method of plaque formation by vaccinia, Newcastle disease (NDV) and western equine encephalomyelitis (WEE) viruses. Of the ten test derivatives, three 2-carboxymethylmercapto-4-amino-5-(chlorphenyl)-pyrimidines inhibited multiplication of vaccinia virus. Four phenylcytosines were found to inhibit WEE virus. No substance selectively inhibited the formation of plaques by NDV. 2-carboxy-methylmercapto-4-amino-5-(p-chlorphenyl)pyrimidine (CACP) was highly selective against vaccinia virus; in membrane cultures its toxic concentration was 15 times higher than its virus inhibiting concentration.

Thermosensitivity as a genetic marker of polioviruses. Z. JANDA, Institute of Sera and Vaccines, Prague

The influence of heat on inactivation of polioviruses was studied. Inactivation was carried out in a water bath at $50^{\circ} \mathrm{C}$. Specimens collected at given intervals were cooled and stored until required at $-30^{\circ} \mathrm{C}$. The effect of different $\mathrm{pH}$ on the rate and degree of inactivation of virus strains was studied. In order to determine whether the use of heat inactivation as a genetic marker was suitable, the relationship between thermosensitivity and neurovirulence and the stability of the t-character after passaging the virus in tissue cultures and in the human aliment. ary tract were studied.

Antigenic stability of polioviruses. V. VonKa, Institute of Sera and Vaccines, Prague

The development of serological methods ruekes it relatively easy to detect differences between polioviruses belonging to the same antigenic type. One of the important oriteria for the differentiation of polioviruses is the use of the "antigenic marker", which is dependent on stability of intratype antigenic character. The author studied the antigenic relationship between LSc $2 a b$ virus and the parental Mahoney virus, the in vitro serological properties of variants of LSc 2ab virus, the stability of intratype antigenic character after passage of the virubes in the human alimentary tract and the antigenic relationships of some serologically different variants. of LSc $2 \mathrm{ab}$ and Mahoney virus.

Changes in tissue culture cells caused by virus strains isolated from pig lungs. V. PuEva, Institute of Veterinary Medicine, Brno

Strains of viruses isolated from pulmonary lesions in sucking-pigs or weaned piglets were used. Isolation was carried out in pig lung or kidney tissue cultures and in chick embryos. The effect of these strains was compared with the effect of haemagglutinating strains of the influenza type. The tissue cultures were set up in test-tubes in which cut slides and cover-slips were placed. The morphological characteristics and development of the tissue culture and changes caused by the viruses were studied. A cytopathogenic effect of a degenerative character developed within $24-48$ hours. The attacked cells contracted and later adhered to one another by means of thin processes. The cytoplasm was vacuolated and consisted of coarse granules. The nuclear membrane contraeted, the chromatin clumped together and the nucleus became pyknotic.

Study of changes in the properties of a strain of Infl. suis virus during adaptation to tissue cultures. D. VĚžnIKová, Institute of Veterinary Medicine, Brno-Medlánky

The sensitivity of different types of tissue (pig kidney, calf and monkey kidney, amnion and HeLa cells) to some strains of Infl. suis virus was compared. A cytopathogenic effect and haemagglutinin formation were found in all the given tissues except HeLa cells. In monkey kidneys and amnion, the cytopathogenic effect and haemagglutinin formation were lost after the second passage. The most suitable tissues for multiplication of Infl. suis virus were calf and pig kidney. The process of adaptation of some strains of Infl. suis virus in tissue cultures and pig kidneys was studied. Strains adapted to chick embryos and freshly isolated strains were used. Only some of the strains became sufficiently adapted to form a constant cytopathogenic effect, while in the other strains the extent of the cytopathogenic effect gradually diminished. During adaptation, certain apparently constant biological properties underwent marked changes in some of the strains.

Experimental erythromycin therapy of staphylococcal inflammation of the lidneys. J. SCHIN DLER, Institute of Medical Microbiology and Immunology, Charles University, Prague

Erythromycin was administered continuously for six days. The dose and interval were based on determination of the amount of erythromycin in the kidney parenchyma and serum. When administered from the fifth day after infection, erythromycin did not influence the course of the infectious process. The number of staphylococci in the tissue and the 
incidence of abscesses remained practically the same. If erythromycin infltrated into the tissues within 48 hours after infection, i.e. before abscesses began to be formed, proliferation of the staphylococci was not inhibited for another 24 hours, but no abscesses were formed. The kidney tissue was rapidly cleared and the number of staphylocoeci decreased.

Immunological demonstration of antigens in a toxic complex produced in broth cultures of Cor. pyogenes hominis. A. Souð̌Ek, A. Souð̌ková and F. Pato ̌ka, Institute of Medical Microbiology and Immunology, Charles University, Prague.

As reported in previous papers, filtrate of broth cultures of human strains of $C$. pyogenes and $C$. haemolyticum has a dermonecrotic and lethal effect in vivo (rabbit and guinea-pig), while in vitro it inhibits staphylococcal $\alpha-\beta$ haemolysis and causes haemolysis. It was also demonstrated that all this activity of the filtrate was neutralized by rabbit hyperimmune serum, showing that it evidently contains a number of toxic or otherwise biologically active antigens. Immune sera were prepared by immunizing rabbits with whole filtrate and with filtrate partly purified by adsorption to erythrocytes. In vitro and in vivo methods were used for immunological and toxicological analysis, including neutralization of haemolysin and of the inhibitor of staphylococcal haemolysis, precipitation of whole and purified filtrate (or of the growing culture) in agar by the method of Ouchterlony and Oakley. Neutralization of the dermonecrotic effect and partial neutralization of the lethal effect was tested in vivo in rabbits. Active filtrate gave one or two precipitation lines with absorbed serum. With the strain $C$. pyogenes hominis, the serum gave two precipitation lines, one of which was also found with strains of $C$. pyogenes bovis. A laboratory standard was determined for indirect titration and the other sera were measured against this. The tests demonstrated that the filtrate contained at least two different antigens, one of which had hesemolytic activity, while the other possessed dormonectrotic activity and was adsorbed to erythrocytes.

Attempt to adapt Pasteurella multocida to Rana esculenta, $R$. temporaria and Bufo vididis. Z. MALfk, Bioveta, Nitra

As part of a series of experiments aimed at obtaining a non-pathogenic variant of $P$. multooida, attempts were made to adapt it to frogs, which are known not to be susceptible to this microorganism. When a strain passaged in golden hamsters was used and hydrocortisone was administered simultaneously, infection and death from pasteurellosis were indueed in both species of frogs. Toads (B. viridis) survived, however, and $P$. multocida could not be isolated from them. A atrain of $P$. multooida recovered from infected frogs after death was passaged further in both species up to the 13th passage, administering hydrocortisone at the same time. Unless hydrocortisone was administered, no discernible disturbance of the frogs' state of health occurred in any of the passages. The percentage of deaths increased with the number of passages and in the 13th passage $60 \%$ of infected frogs treated with hydrocortisone died. The test strain lost none of its pathogenicity for white mice, rabbits and fowls during passaging.

\section{Influence of microorganisms in the reproduction} process in animals. J. KAzDA, Central Breeding Station, Brno branch

When studying the influence of microorganisms occurring most frequently in the sex orgens in animals, it was found that some had a spermicidal effect, while others had a favourable effect, by helping to preserve the spermatozoa. At $39^{\circ} \mathrm{C}$ Escherichia coli, $E$, freundi, Proteus vulgaris, $P$. mirabilis, Corynebacterium pyogenes and $C$, viridans (differentiated according to Bratke, 1954) had a highly epermicidal effeet. Corynebacterium opecies (with the exception of $C$. pyogenes and $C$. viridans was found to increase the number of progressively motile spermatozoa during storage at $39^{\circ} \mathrm{C}$. Experiments were carried out with bovine spermatozoa and with strains isolated from the sex organs of these animals. Anomalies were found in the diagnosis, particularly in the presence of urease and indol production, indicating that these microorganisms are adapted to the environment in which they live mostly as saprophytes.

Use of the fluorescent antibody method for the detection of pathogens in gynaecology. Z. V.žnt́k, D. VĚŽNfKOVÁ and $K$. Ż́K, Institute of Veterinary Medicine and Second Maternity and Gynaeeology Clinic, Brno

Methods of detecting brucellae in the genitalia of guinea-piga were studied. The guinea-pigs were infected intraperitoneally and intramuseularly. For estimation of the standard amount of pathogenic microorganisms, the suspension was diluted accord. ing to MeFarland's nephelometric standard No. 4. Material from the guinea-pige was examined by the fluorescent antibody method. Imears from peritoneal punetures, imprints from the peritoneum, liver, spleen, lymph nodes and endometrium, prepared after the guinea-pigs were killed, and smears obtained by abrasion of the uterine cavity, were stained with a conjugated stain obtained by the method of Coons and Cherry. Specifie fluores. cenee was demonstrated in the tissues and in smears of the abraded parts of the endometrium of experimentally infeeted animals. Control negative and inhibited material had only minimal (nonspecific, tissue) fluorescence. The use of fluorescent antibodies in infections of the genital tract will be studied 
further. From the results obtained hitherto it can be concluded that fluorescent antibodies undoubtedly mean an advance in clinical diagnostics.

Survival of Brucella abortus and Brucella suis in meat products. J. SvoBoDA and M. DoBES, Faculty of Veterinary Medicine, Agricultural College, Brno

The authors determined the effect of technological processes on $B$. abortus Bang and $B$. suis in meat products, different methods of isolating brucellae from this strongly saprophyte-contaminated medium and the applicability of allergodiagnostics in the biological demonstration of brucellae in guinea-pigs. The results showed that both species of Brucella survived the technological process in products smoked at low temperatures (Bologna sausage). CTN agar with polymyxin and bacitracin also proved suitable for the isolation of brucellae from strongly contaminated products $\left(10^{7}-10^{5}\right)$. Falze's Brucellar allergen $F$ was likewise satisfactory in the biological demonstration of brucellae in experimental guinea-pigs, the reaction up to $1 \mathrm{~mm}$. being negative, from $1.1-1.9 \mathrm{~mm}$. dubious and over $2 \mathrm{~mm}$. reliably positive.

Modified virus of Aujeszky's disease suitable for immunization purposes. A. ZUTFA, Bioveta, Nitra

The virus of Aujeszky's disease was passaged in chick embryo cell (CEC) cultures. After 200 passages in cultures cultivated in Parker medium at $36^{\circ} \mathrm{C}$, the virus underwent another 200 passages in cultures in Earl and Parker's medium at the same temperature, or 100 passages at $30^{\circ} \mathrm{C}$. The virulence of the virus for mice, guinea-pigs and rabbits did not alter in the course of 400 passages in CEC cultures; the only change was in the clinical manifestations of the disease in these animals. After 200 CEC passages in Parker's medium and 50 in Earl's medium, or after 300 CEC passages in Parker's medium, however, the virus lost its neuropathogenicity for four-woek-old lambs (the youngest tested). After 400 CEC passages (200 in Parkers's medium and 200 in Earl's medium) the virus was harmless for sheep and five-day-old calves when administered intramuscularly, although after 400 CEC passages in Parker's medium the virus was 50\% lethal for inoculated sheep. The decisive factors in modification of the virus were the number of passages and the composition of the culture medium; the temperature had no significant influence. The administration of a single dose of modified virus to pigs, calves and sheep induced the formation of low VN antibody, titres (2-16), which were raised $(32-512)$ by revaccination. Progressive passage of the virus in CEC lowered its ability to induce antibody formation in inoculated animals.

Development of antibody formation against Influenza suis virus in piglets during the first weeks of life. J. MENŜ́k and D. ŽknnföEk, Institute of Veterinary Medicine, Brno-Medlánky

In model experiments the authors studied the dynamics of colostrum antibodies, serum proteins and the antibody response of suckling-pigs to Infl. suis antigen administered at different intervals after birth. Even large doses of antigen failed to influence the decrease in colostrum antibodies in piglets from serologically positive mothers and a weak or negative antibody response was still registered at various intervals after they had completely disappeared. In piglets from serologically negative mothers infected with Infl. suis virus within four days after birth, significant $\mathrm{HI}$ and $\mathrm{VN}$ antibody titres were recorded 4-12 days after infection, i.e. during the first two weoks of life. The dynamics of antibodies formed in this early phase of postnatal development are reminiscent of the dynamics of colostrum antibodies, particularly as they always disappear within the first $6-10$ weeks of life. The same doses of antigen administered to adult pigs induce a different antibody response and the antibodies persist for several months and often for life. The decrease in colostral $\gamma$ globulin in the serum of calves was influenced by infection with Infl. suis virus and the HI antibody level was not directly related to the gamma globulin level. The absence of antibodies against Infl. suis virus in pigs can be attributed to inhibition of antibody formation through constant contact of the pigs with relatively small amounts of antigen while colostral immunity still persisted and after it had disappeared, when colostrum antibodies could no longer be demonstrated by the usual serological methods.

Study of immunity mechanisms in brucellosis. J. Mrierová and D. Vackáḱová, Institute of Sera and Vaccines, Prague

The experiments follow on from a previous report (1961) on an extracellular protective antigen released by a live culture of the vaccine strain BA-19-Zdrodovski into liquid culture medium under specific kinetic cultivation conditions. In further experiments this protective antigen, prepared in vitro, was compared with a similar protective factor contained in sterile peritoneal fluid from guinea-pigs inoculated intraperitoneally with vaccine strain BA-19. An active protection test showed that both the in vitro and the in vivo preparation protected mice and guinea-pigs against virulent infection. The values of this protection were comparable with the activity of live vaccine prepared from this strain and tested under the same experimental conditions. It was found that the antigenic character of the extracellular protective antigen was difficult to demonstrate by the usual serological methods. Serum and sterile exudate from mice and guinea-pigs in a state of active protection after immunization with extracellular protective antigen had a significant protective effect, however (demoristrated in a passive protection test in mice). Serum and exudate 
from animals immunized with live vaccine had similar properties. On the basis of the similarity of the activity of the above preparations, the hypothesis is expressed that contact of the live vaccine with the macroorganism gives rise to a similar extracellular protective component, which is an important factor in its protective effect. The possibilities for the practical application of these findings are now being studied.

Conditions for antibody formation by spleen fragments in vitro. C. JOHN and P. CoRvazIER, Institute of Medical Microbiology and Immunology, Charles University, Prague

Chick spleen fragments cultivated in vitro form antiphage (anti- $T_{2}$ ) antibodies if the antigen is administered to the chick in vivo (intravenously) at least 24 (and preferably 28) hours before removing and cultivating the spleen. If the chick is immunized with a single dose 48 hours before removing and cultivating the spleen, antibodies, are released into the culture medium after the same interval, in large quantities. A small amount of antibody can also be demonstrated in the medium on the seventh day of cultivation. If the antigen is administered to the chicks intravenously, the spleen fragments still release antibodies into the culture medium even if the in vitro culture is not set up until 23 days after immunization. Antibody formation is further stimulated by reimmunizing the chicks 28 hours before removing the spleen. If the antigen is admin. istered intraperitoneally, no primary antibody response can be demonstrated in an in vitro culture set up after 23 days. After intraperitoneal reim. munization, a secondary antibody response can be detected in vitro. The hypothesis is expressed that a complete antibody formation cycle takes place in vitro only if contact of the antigen with microphages is ensured before mixing with cells capable of antibody synthesis. When studying antibody formation by isolated rabbit spleen cells transferred to the peritoneal cavity of immature young, it was found that macrophage digestion of antigenic material (phage $T_{2}$ ) did not stimulate antibody formation.

Adjuvant effect of sodium polymethacrylate in immunization. J. SCHIN DLER and R. NABÁKOVÁ, Institute of Medical Microbiology and Immunology, Charles University, Prague
The addition of sodium polymethacrylate to inactivated corpuscular Brucella abortus $\mathrm{BrO}$ antigen enhances agglutinin production in rabbits (demonstrable up to the 35th day). This result is discussed from the aspect of the selection of useful adjuvants and of the actual property of adjuvant effect.

Study of certain factors of nonspecific immunity in preserved blood-properdin, complement, bactericidal activity. A. JIRsÁkovA, Faculty Transfugion Station, Prague

In A.C.D.-preserved blood, the properdin and complement level was determined and its decrease during storage in a refrigerator at $+4^{\circ} \mathrm{C}$ for two weeks was studied. The results were evaluated by $\mathrm{a}$ paired $t$-test and the decrease was found to be statistically significant. The bactericidal activity. of preserved blood and changes during storage were evaluated by determining the bactericidal index. The results were also evaluated by a paired $t$-test and a statistically significant decrease was found (during two weeks bactericidal activity fell to $55 \%$ of the initial level). Changes in properdin and bactericidal activity were compared and attention was drawn to the properties of both these immunological factors.

Heterohaemagglutination in infectious hepatitis. K. SrMEK and J. HoRECKA, Departmept of Microbiology, Faculty of Medicine, Purkynè University, Brno

The authors report the results of a heterohaemagglutination reaction with serum from patients with a diagnosis of infectious hepatitis and fowl blood cells. The heteroagglutination reaction was positive in $76.2 \%$ of these patients. Serum from patents with other liver diseases gave $22.5 \%$ positive results, while in healthy subjects or patients with other than liver diseases the number of positive results was $6.1 \%$. The agglutination titres tended to decrease with the time from the onset of the disease. Because of its simplicity and the ease with which it can be carried out, the authors recommend this reaction as one of the diagnostic methods for infectious hepetitis and as an auxiliary method in the differential diagriosis of infectious hepatitis and other liver diseases. 